
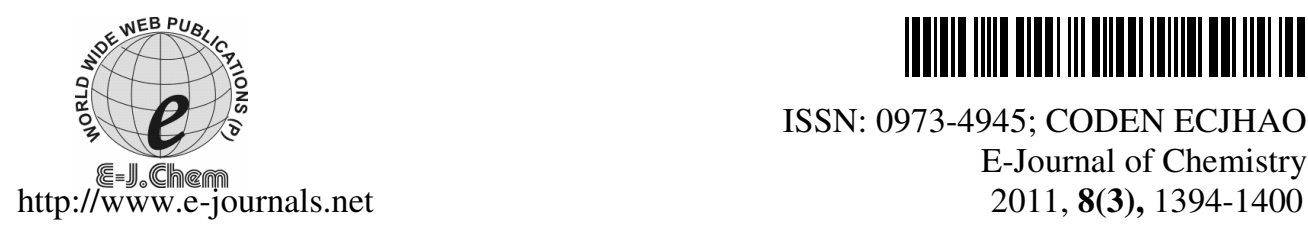

ISSN: 0973-4945; CODEN ECJHAO

E-Journal of Chemistry

2011, 8(3), 1394-1400

\title{
Design and Synthesis of Small Molecules Based on a Substructural Analysis of the Histone Deacetylase Inhibitors TSA and SAHA
}

\author{
LYNDA EKOU*, TCHIRIOUA EKOU, JAVIER GARCIA ${ }^{\S}$, \\ ISABELLE OPALINSKI $^{\S}$ and JEAN PIERRE GESSON ${ }^{\S}$
}

Université d'Abobo-Adjamé

Laboratoire de Thermodynamique et de Physico Chimie du Milieu

02 BP 801 Abidjan 02, Côte d'Ivoire

${ }^{\S}$ Université de Poitiers, UMR-CNRS 6514,

Laboratoire de Synthèse et Réactivité des Substances Naturelles

40 Avenue du Recteur Pineau, 86022 Poitiers Cedex, France

ekou_lynda@yahoo.fr

Received 30 June 2010; Revised 3 December 2010; Accepted 8 January 2011

\begin{abstract}
Inhibitors of histone deacetylases (HDACs) are patent inducers of differentiation and bear considerable potential as drugs for chemoprevention and treatment of cancer. In this paper, we have investigated three synthetic, inhibitors A1a,b, A2a. Analogue hybrid trichostatine A (TSA), suberoylanilide hydroxamic acid SAHA, in order to seek new histone deacetylases (HDACs) inhibitors.
\end{abstract}

Keywords: Histone deacetylase, Hydroxamic acids, Enzyme inhibitors, Substructural analysis.

\section{Introduction}

Histone deacetylases (HDACs) and histone acetyl transferases (HATs) are known to play an important role in the regulation of gene expression ${ }^{1,2}$. HATs mediate hyperacetylation of positively charged lysine residues in the $N$-terminal tail of core histone and loosen the histone-DNA binding. As a result, activation of genes transcription can occur. In contrast HDACs catalyse deacetylation of the acetyl $\varepsilon$ amino group residues and lead to the tight histone-DNA binding ${ }^{3}$. In this case, the access to transcription factors is restricted. These enzymes correlate with cell cycle progression, differentiation and apoptosis ${ }^{4}$ and their deregulation is associated with tumorogenisis ${ }^{5}$. 
HDACs inhibitors have demonstrated potential for the prevention and treatment of cancer in numerous cell culture ${ }^{6,7}$ and animals models ${ }^{8}$. HDACs have emerged as an attractive target for new anticancer drugs and there is a great demand for new inhibitors ${ }^{9,10}$.

The well known histone deacetylase inhibitor trichostatine A $(\mathrm{TSA})^{11,12}(\mathbf{1})$, a natural product and other synthetic compounds such as suberoylanilide hydroxamic acid (SAHA) ${ }^{13-15}$ (2) and analogues ${ }^{16}$, oxamflatin ${ }^{17}(3)$, or MS-275 ${ }^{18}(4)$ possess potent antitumor effect in vivo in tumor-bearing animals ${ }^{19}$ and some of them are currently in phase I or phase I / phase II clinical trials $^{20-24}$ (Figure 1).<smiles>CNc1ccc(C(=O)C(C)/C=C(C)/C=C/C(=O)NO)cc1</smiles>

Trichostatine A (1)<smiles>O=C(/C=C/C#Cc1cccc(NS(=O)(=O)c2ccccc2)c1)NO</smiles>

Oxamflatin (3)<smiles>O=C(CCCCCCC(=O)Nc1ccccc1)NO</smiles>

SAHA (2)<smiles>Nc1ccccc1NC(=O)c1ccc(CNC(=O)OCc2cccnc2)cc1</smiles>

MS-275 (4)

Figure 1. Structures of known HDAC inhibitors

These compounds consist of a hydrophobic scaffold with a spacer that is attached to a functional group which can interact with zinc ion present in the active site pocket ${ }^{25-26}$. Compounds containing hydroxamic acid as a functional group are reported as the most potent inhibitors for HDACs ${ }^{16}$.

\section{Experimental}

Nuclear magnetic resonance spectra $\left({ }^{1} \mathrm{H}\right.$ NMR and ${ }^{13} \mathrm{C}$ NMR) were recorded on a Bruker 300 and $75 \mathrm{MHz}$. Flash column chromatography was performed using MACHEREYNAGEL silica gel $60(15-40 \mu \mathrm{m})$ as the stationary phase. All reactions were run under a positive pressure of nitrogen unless otherwise stated.

\section{[1-(4-Methoxy-phenylcarbamoyl)-ethyl]-carbamic acid tert-butyl ester (7a)}

To a solution of $N$-(tert-butoxycarbonyl)- $L$-alanine 5 (32.5 mmol, 1 eq.) and aniline 6a (32.5 mmol, 1 eq.) in dimethylformamide (DMF) $(80 \mathrm{~mL})$ were added portion wise of diphenylphosphorylazide (DPPA) $(7.7 \mathrm{~mL}, 33.7 \mathrm{mmol})$ and triethylamine (TEA) $(9.70 \mathrm{~mL}, 69.8 \mathrm{mmol})$ at $0{ }^{\circ} \mathrm{C}$. The stirring was continued at room temperature overnight. dimethylformamide was removed under reduced pressure. The residue was purified by flash chromatography (Ethyl acetate / Petroleum ether, 40/60) to give 7a (90\%, white solid). ${ }^{1} \mathrm{H}$ NMR $\left(\mathrm{CDCl}_{3}\right) \delta \mathrm{ppm} 1.44(\mathrm{~m}, 12 \mathrm{H}), 3.77(\mathrm{~s}, 3 \mathrm{H}), 4.38$ $(\mathrm{sl}, 1 \mathrm{H}), 5.38(\mathrm{~d}, J=7.6 \mathrm{~Hz}, 1 \mathrm{H}), 6.84(\mathrm{~d}, J=8.7 \mathrm{~Hz}, 2 \mathrm{H}), 7.40(\mathrm{~d}, J=8.7 \mathrm{~Hz}, 2 \mathrm{H})$, $8.59(\mathrm{~s}, 1 \mathrm{H}) .{ }^{13} \mathrm{C} \mathrm{NMR}\left(\mathrm{CDCl}_{3}\right) \delta \mathrm{ppm} \mathrm{18.4,} \mathrm{28.7,} \mathrm{50.9,} \mathrm{55.8,} \mathrm{80.7,} \mathrm{114.3,} \mathrm{122.0,}$ 131.3, 156.5, 171.3. 
[1-(4-Dimethylamino-phenylcarbamoyl)-ethyl]-carbamic acid tert-butyl ester (7b) White solid (yield 78\%). ${ }^{1} \mathrm{H}$ NMR $\left(\mathrm{CDCl}_{3}\right) \delta \mathrm{ppm} 1.45(\mathrm{~m}, 12 \mathrm{H}), 2.83(\mathrm{~s}, 6 \mathrm{H}), 4.38(\mathrm{sl}, 1 \mathrm{H})$, $5.15(\mathrm{sl}, 1 \mathrm{H}), 6.84(\mathrm{~d}, J=9.1 \mathrm{~Hz}, 2 \mathrm{H}), 7.40(\mathrm{~d}, J=9.1 \mathrm{~Hz}, 2 \mathrm{H}), 8.13(\mathrm{sl}, 1 \mathrm{H}) .{ }^{13} \mathrm{C} \mathrm{NMR}$ $\left(\mathrm{CDCl}_{3}\right) \delta \mathrm{ppm} 18.6,28.7,41.3,50.9,80.5,113.3,122.1,128.0,148.3,156.3,171.1$.

\section{2-Amino-N-(4-methoxy-phenyl)-propionamide ( 8 a)}

The protected compound $7 \mathbf{a}$ was dissolved in trifluoroacetic acid (TFA) (29 mL) at room temperature and kept for $2 \mathrm{~h}$. The mixture was evaporated under reduced pressure. The residue was neutralised with $\mathrm{Na}_{2} \mathrm{CO}_{3}$ and consecutively extracted with ethyl acetate. The organic layer was washed with water then brine, dried over $\mathrm{Na}_{2} \mathrm{SO}_{4}$ and concentrated in vacuo. Purification by flash chromatography $\left(\mathrm{CH}_{2} \mathrm{Cl}_{2} / \mathrm{MeOH}, 90 / 10\right)$ gave 8a (white solid). ${ }^{1} \mathrm{H}$ NMR $\left(\mathrm{CDCl}_{3}\right) \delta \mathrm{ppm} 1.33(\mathrm{~d}, J=7.0 \mathrm{~Hz}, 3 \mathrm{H}), 3.45(\mathrm{sl}, 2 \mathrm{H}), 3.77(\mathrm{~m}, 4 \mathrm{H}), 6.89$

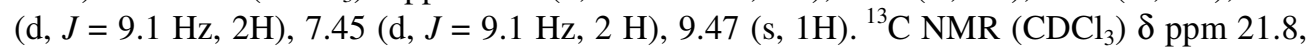
51.2, 55.7, 114.4, 122.4, 131.7, 132.8, 157.6.

\section{2-Amino-N-(4-dimethylamino-phenyl)-propionamide $(\mathbf{8 b})$}

White solid. ${ }^{1} \mathrm{H}$ NMR (DMSO) $\delta$ ppm $1.41(\mathrm{~d}, J=7 \mathrm{~Hz}, 3 \mathrm{H}), 2.85$ (s, 6H), 3.91 (q, $J=7 \mathrm{~Hz}$, $1 \mathrm{H}), 6.73(\mathrm{~d}, J=9.1 \mathrm{~Hz}, 2 \mathrm{H}), 7.45(\mathrm{~d}, J=9.1 \mathrm{~Hz}, 2 \mathrm{H}), 8.00(\mathrm{sl}, 3 \mathrm{H}) .{ }^{13} \mathrm{C}$ NMR (DMSO) $\delta$ ppm 17.6, 40.7, 49.1, 112.9, 121.1, 128.0, 167.6.

\section{4-[1-(4-Methoxy-phenylcarbamoyl)-ethylamino]-pent-4-enoic acid (9a)}

To a solution of amine $\mathbf{8 a}$ in dimethylformamide $(15 \mathrm{~mL})$ were added succinic anhydride ( $8.81 \mathrm{mmol}, 1$ eq.) and triethylamine $(1.23 \mathrm{~mL}, 8.81 \mathrm{mmol})$. The mixture was stirred for $4 \mathrm{~h}$ at room temperature. After evaporation of organic solvents, the residue was purified by flash chromatography using $\left(\mathrm{CH}_{2} \mathrm{Cl}_{2} / \mathrm{MeOH}, 90 / 10\right)$ to give compound $9 \mathrm{a}\left(93 \%\right.$, white solid). ${ }^{1} \mathrm{H}$ NMR (DMSO) $\delta$ ppm $1.34(\mathrm{~d}, J=6.7 \mathrm{~Hz}, 3 \mathrm{H}), 2.49(\mathrm{~m}, 4 \mathrm{H}), 3.79(\mathrm{~s}, 3 \mathrm{H}), 4.44(\mathrm{~m}, 1 \mathrm{H})$, $6.95(\mathrm{~d}, J=8.5 \mathrm{~Hz}, 2 \mathrm{H}), 7.59(\mathrm{~d}, J=8.5 \mathrm{~Hz}, 2 \mathrm{H}), 8.30(\mathrm{~d}, J=6.9 \mathrm{~Hz}, 1 \mathrm{H}), 10.00(\mathrm{~s}, 1 \mathrm{H})$, $12.31(\mathrm{sl}, 1 \mathrm{H}) .{ }^{13} \mathrm{C}$ NMR (DMSO) $\delta \mathrm{ppm} \mathrm{18.5,} \mathrm{29.4,} \mathrm{30.1,} \mathrm{49.2,} \mathrm{55.4,} \mathrm{114.1,} \mathrm{121.1,} \mathrm{130.4,}$ 155.5, 171.1, 171.3, 174.3.

\section{4-[1-(4-Dimethylamino-phenylcarbamoyl)-ethylamino]-pent-4-enoic acid (9b)}

(61\%, White solide). ${ }^{1} \mathrm{H}$ NMR (DMSO) $\delta \mathrm{ppm} 1.41(\mathrm{~d}, J=7.0 \mathrm{~Hz}, 3 \mathrm{H}), 2.74(\mathrm{~m}, 4 \mathrm{H}), 2.97$ $(\mathrm{s}, 6 \mathrm{H}), 4.50(\mathrm{~m}, 1 \mathrm{H}), 6.62(\mathrm{~d}, J=8.9 \mathrm{~Hz}, 2 \mathrm{H}), 7.59(\mathrm{~d}, J=8.9 \mathrm{~Hz}, 2 \mathrm{H}), 8.35(\mathrm{~d}, J=7.32$ $\mathrm{Hz}, 1 \mathrm{H}), 9.71(\mathrm{~s}, 1 \mathrm{H}), 12.20(\mathrm{sl}, 1 \mathrm{H})$.

N-Hydroxy-N-[1-(4-methoxy-phenylcarbamoyl)-ethyl]-succinamide (A1-a)

To acid 9a (200 mg, $0.68 \mathrm{mmol}), \quad$ 1-[3(dimethylamino)propyl]-3-ethyl-carboimide hydrochloride] (EDC) (196.1 mg, $1.02 \mathrm{mmol}), N$-hydroxybenzotriazole (HOBT) (119.9 mg, $0.89 \mathrm{mmol}$ ) was reacted in dimethylformamide for $1 \mathrm{~h} . \mathrm{NH}_{2} \mathrm{OBn} . \mathrm{HCl}(108.9 \mathrm{mg}, 0.68$ mmol), triethylamine $(0.10 \mathrm{~mL}, 0.68 \mathrm{mmol})$ were added and the mixture was stirred for 2 days. DMF was removed under reduced pressure. The residue was purified by flash chromatography using $\mathrm{CH}_{2} \mathrm{Cl}_{2} / \mathrm{MeOH}, \quad 95 / 5$ to give $N$-benzyloxy- $N$-[1-4-metoxyphenylcarbamoyl)-ethyl]-succinamide (white solid, 48\%).

${ }^{1} \mathrm{H}$ NMR (DMSO-d $\left.{ }_{6}\right) \delta$ ppm $1.47(\mathrm{~d}, J=7.1 \mathrm{~Hz}, 3 \mathrm{H}), 2.43(\mathrm{t}, J=6.4 \mathrm{~Hz}, 2 \mathrm{H}), 2.62$ (t, $J=6.4 \mathrm{~Hz}, 2 \mathrm{H}), 3.89(\mathrm{~s}, 3 \mathrm{H}), 4.56(\mathrm{~m}, 3 \mathrm{H}), 4.96(\mathrm{~s}, 2 \mathrm{H}), 7.02(\mathrm{~d}, J=9.0 \mathrm{~Hz}, 2 \mathrm{H}), 7.56$ $(\mathrm{m}, 5 \mathrm{H}), 7.75(\mathrm{~d}, J=9.0 \mathrm{~Hz}, 2 \mathrm{H}), 8.46(\mathrm{~d}, J=7.3 \mathrm{~Hz}, 1 \mathrm{H}), 9.90(\mathrm{~s}, 1 \mathrm{H}), 11.28(\mathrm{~s}, 1 \mathrm{H}) .{ }^{13} \mathrm{C}$ NMR (DMSO-d $\left.{ }_{6}\right) \delta$ ppm 18.4, 30.5, 28.0, 49.2, 55.4, 77.1, 114.0, 121.1, 128.5, 129.0, 132.4, 155.5, 169.3, 171.2. 
The $N$-benzyl precursor $(0.66 \mathrm{mmol})$ was dissolved in $\mathrm{MeOH}(10 \mathrm{~mL}), 10 \% \mathrm{Pd}-\mathrm{C}$ was added and the solution was shaken under $\mathrm{H}_{2}$. After $4 \mathrm{~h}$ the mixture was filtered through celite and the filtrate was evaporated. The hydroxamic acid A1-a was isolated after purification of small sample on preparative plates (qt, withe solid). ${ }^{1} \mathrm{H}$ NMR (DMSO-d $\left.{ }_{6}\right) \delta$ ppm 1.35 $(\mathrm{d}, J=7.1 \mathrm{~Hz}, 3 \mathrm{H}), 2.29(\mathrm{t}, J=6.7 \mathrm{~Hz}, 2 \mathrm{H}), 2.45(\mathrm{t}, J=6.7 \mathrm{~Hz}, 2 \mathrm{H}), 3.87(\mathrm{~s}, 3 \mathrm{H}), 4.45$ $(\mathrm{m}, 1 \mathrm{H}), 6.95(\mathrm{~d}, 2 \mathrm{H}, J=9.0 \mathrm{~Hz}, 2 \mathrm{H}), 7.63(\mathrm{~d}, J=9.0 \mathrm{~Hz}, 2 \mathrm{H}), 8.30(\mathrm{~d}, J=7.3 \mathrm{~Hz}, 1 \mathrm{H})$, $9.18(\mathrm{~s}, 1 \mathrm{H}), 9.83(\mathrm{~s}, 1 \mathrm{H}), 10.50(\mathrm{~s}, 1 \mathrm{H}) .{ }^{13} \mathrm{C} \mathrm{NMR}\left(\mathrm{DMSO}_{-} \mathrm{d}_{6}\right) \delta \mathrm{ppm} 18.4,30.8,28.0,49.1$, $55.4,114.0,121.1,136.4,155.5,168.9,171.2$.

\section{$N$-[1-(4-Dimethylamino-phenylcarbamoyl)-ethyl]-N'-hydroxy-succinamide (A1-b)}

To a solution of acid $9 b\left(0.5\right.$ mmol, 1 eq.) and $\mathrm{NH}_{2} \mathrm{OBn} . \mathrm{HCl}(0.159$ g, 1 eq.) in dimethylformamide $(2 \mathrm{~mL})$ diphenylphosphorylazide $(0.12 \mathrm{~mL}, 0.55 \mathrm{mmol})$ and triethylamine $(0.14 \mathrm{~mL}, 1 \mathrm{mmol})$ were added portion wise at $0{ }^{\circ} \mathrm{C}$. The stirring was continued at room temperature for $1 \mathrm{~h}$ and $30 \mathrm{~min}$. Dimethylformamide was removed under reduced pressure. The residue was purified by flash chromatography using $\mathrm{CH}_{2} \mathrm{Cl}_{2} / \mathrm{MeOH}$, 90/10 to obtain $N$-benzyl- $N$-[1-(4-dimethylamino-phenylcarbamoyl)-ethyl]-succinamide (white solid, 50\%). ${ }^{1} \mathrm{H}$ NMR (DMSO-d $\left.{ }_{6}\right) \delta \mathrm{ppm} 1.27(\mathrm{~m}, 3 \mathrm{H}), 2.31(\mathrm{~m}, 2 \mathrm{H}), 2.42(\mathrm{~m}, 2 \mathrm{H})$, $2.88(\mathrm{~s}, 6 \mathrm{H}), 4.37(\mathrm{~m}, 1 \mathrm{H}), 4.83(\mathrm{~s}, 2 \mathrm{H}), 6.71(\mathrm{~d}, J=8.9 \mathrm{~Hz}, 2 \mathrm{H}), 7.06(\mathrm{~m}, 7 \mathrm{H}), 8.37(\mathrm{~d}, J=$ $7.3 \mathrm{~Hz}, 1 \mathrm{H}), 9.51(\mathrm{~s}, 1 \mathrm{H}), 11.23(\mathrm{~s}, 1 \mathrm{H})$.

The $N$-benzyl derivative was subjected to hydrogenolysis with $10 \% \mathrm{Pd}-\mathrm{C}$ in $\mathrm{MeOH}$ in the same way as for A1-a to give A1-b (73\%, white solid). ${ }^{1} \mathrm{H}$ NMR (DMSO-d ${ }_{6}$ ) $\delta$ ppm 1.10 $(\mathrm{m}, 3 \mathrm{H}), 2.05(\mathrm{~m}, 2 \mathrm{H}), 2.21(\mathrm{~m}, 2 \mathrm{H}), 2.66(\mathrm{~s}, 6 \mathrm{H}), 4.16(\mathrm{~m}, 1 \mathrm{H}), 6.51(\mathrm{~d}, J=9 \mathrm{~Hz}, 2 \mathrm{H}), 7.28$ $(\mathrm{d}, J=9 \mathrm{~Hz}, 2 \mathrm{H}), 8.04(\mathrm{~d}, J=7.32 \mathrm{~Hz}, 1 \mathrm{H}), 8.56(\mathrm{sl}, 1 \mathrm{H}), 9.43(\mathrm{~s}, 1 \mathrm{H}), 10.26(\mathrm{~s}, 1 \mathrm{H})$.

\section{4-Oxo-pent-2enoic acid [1-(4-methoxy-phenylcarbamoyl)-ethyl]-amide (10)}

To a solution of mono ethyl ester fumaric acid (373 mg, 2,6 mmol) in DMF (7 mL) 1[3(dimethylamino)propyl]-3-ethyl-carboimide hydrochloride $(743.8 \mathrm{mg}, 3.9 \mathrm{mmol})$ and $\mathrm{N}$ hydroxybenzotriazole $(455 \mathrm{mg}, 3.37 \mathrm{mmol}$ ) were added. The mixture was stirred at room temperature for $1 \mathrm{~h}$. Amine $\mathbf{8 a}(500 \mathrm{mg}, 2.57 \mathrm{mmol})$ was added followed by triethylamine $(0.36 \mathrm{~mL}, 2.57 \mathrm{mmoL})$. The mixture was stirred overnight, then the solvent was evaporated. The residue was purified by flash chromatography using EtOAc/EP, 50/50 to obtain compound 10 (white solid, 49\%). ${ }^{1} \mathrm{H}$ NMR $\left(\right.$ DMSO-d $\left._{6}\right) \delta$ ppm $1.41(\mathrm{t}, J=7.1 \mathrm{~Hz}, 3 \mathrm{H}), 1.50$ $(\mathrm{d}, J=7.0 \mathrm{~Hz}, 3 \mathrm{H}), 3.89$ (s, 3H, ), 4.35 (q, $J=7.1 \mathrm{~Hz}, 2 \mathrm{H}), 4.67(\mathrm{~m}, 1 \mathrm{H}), 6.75(\mathrm{~d}, J=15.5$ $\mathrm{Hz}, 1 \mathrm{H}), 7.05(\mathrm{~d}, J=9.0 \mathrm{~Hz}, 2 \mathrm{H}), 7.31(\mathrm{~d}, J=15.5 \mathrm{~Hz}, 1 \mathrm{H}), 7.67(\mathrm{~d}, J=9 \mathrm{~Hz}, 2 \mathrm{H}), 9.06(\mathrm{~d}$, $J=7.3 \mathrm{~Hz}, 1 \mathrm{H}), 10.12(\mathrm{~s}, 1 \mathrm{H}) .{ }^{13} \mathrm{C}$ NMR $\left(\mathrm{DMSO}_{-} \mathrm{d}_{6}\right) \delta \mathrm{ppm} \mathrm{14.3,} \mathrm{18.5,} \mathrm{49.6,} \mathrm{55.4,} \mathrm{61.0,}$ $114.1,121.1,128.9,132.3,137.6,155.6,162.8,165.3,170.5$.

But-2-enodioic acid hydroxyamide[1-(4-methoxy-phenylcarbamoyl)-ethyl]-amide (A2-a)

The ester 10 (130 mg, 0. $41 \mathrm{mmol})$ was dissolved in $\mathrm{MeOH}(40 \mathrm{~mL})$ and an aqueous solution of $\mathrm{LiOH}(16.24 \mathrm{~mL}, 1 \mathrm{M})$ was added. The mixture was stirred for $2 \mathrm{~h}$ at the room temperature. The solution was neutralised with the amberlite resine IRN-77 then, the product was filtered and evaporated to give 3-[1-(4-methoxy-phenylcarbamoyl)-ethylcarbamoyl]acrylic acid (white solid, 57\%), which was used without further purification. ${ }^{1} \mathrm{H}$ NMR $\left(\right.$ DMSO-d $\left._{6}\right) \delta$ ppm $1.38(\mathrm{~d}, J=7 \mathrm{~Hz}, 3 \mathrm{H}), 3.78(\mathrm{~s}, 3 \mathrm{H}), 4.56(\mathrm{~m}, 1 \mathrm{H}), 6.59(\mathrm{~d}, J=15.5 \mathrm{~Hz}, 1 \mathrm{H})$, $6.90(\mathrm{~d}, J=9.0 \mathrm{~Hz}, 2 \mathrm{H}), 7.16(\mathrm{~d}, J=15.5 \mathrm{~Hz}, 1 \mathrm{H}), 7.56(\mathrm{~d}, J=9 \mathrm{~Hz}, 2 \mathrm{H}), 8.9(\mathrm{~d}, J=7.3$ $\mathrm{Hz}, 1 \mathrm{H}), 10.00(\mathrm{~s}, 1 \mathrm{H}), 12.94(\mathrm{sl}, 1 \mathrm{H}) .{ }^{13} \mathrm{C}$ NMR $\left(\mathrm{DMSO}_{-} \mathrm{d}_{6}\right) \delta \mathrm{ppm} \mathrm{18.5,} \mathrm{49.6,} \mathrm{55.5,} \mathrm{114.1,}$ 
$121.1,130.2,132.3,137.1,155.6,163.1,166.7,170.5$. To a solution of acid $(50 \mathrm{mg}$, $0.17 \mathrm{mmol})$ in DMF $(0.5 \mathrm{~mL})$ were added $O$-(bentriazol-1-yl-1, 3, 3)-tetraméthyl-uronium tétrafluoroborate (TBTU) $(54 \mathrm{mg}, 0.17 \mathrm{mmol})$ and triethylamine $(0.05 \mathrm{~mL}, 0.34 \mathrm{mmol})$. The mixture was stirred for $2 \mathrm{~h}$ and then $\mathrm{NH}_{2} \mathrm{OTHP}(0.17 \mathrm{mmol}, 22 \mathrm{mg})$ was added. The mixture was stirred for 5 days at room temperature. dimethylformamide was removed under low pressure, then the crude product was purified using the preparative plate $\left(\mathrm{CH}_{2} \mathrm{Cl}_{2} / \mathrm{MeOH}, 92 / 8\right)$ to give compound A2-a (66\%, withe solid). ${ }^{1} \mathrm{H}$ NMR (DMSO- $\mathrm{d}_{6}$ ) $\delta \mathrm{ppm} 1.26(\mathrm{~d}, J=7.0 \mathrm{~Hz}, 3 \mathrm{H}), 3.65(\mathrm{~s}, 3 \mathrm{H}), 4.43(\mathrm{~m}, 1 \mathrm{H}), 6.47(\mathrm{~d}, J=15.5 \mathrm{~Hz}, 1 \mathrm{H}), 6.82$ $(\mathrm{d}, J=9.0 \mathrm{~Hz}, 2 \mathrm{H}), 6.99(\mathrm{~d}, J=15.5 \mathrm{~Hz}, 1 \mathrm{H}), 7.47(\mathrm{~d}, J=9.0 \mathrm{~Hz}, 2 \mathrm{H}), 8.76(\mathrm{~d}, J=7.3$ $\mathrm{Hz}, 1 \mathrm{H}), 9.86(\mathrm{~s}, 1 \mathrm{H}) .{ }^{13} \mathrm{C} \mathrm{NMR}\left(\mathrm{DMSO}_{6}\right) \delta \mathrm{ppm} \mathrm{18.4,} \mathrm{49.6,55.5,} \mathrm{114.2,} \mathrm{121.2,} \mathrm{130.2,}$ $132.3,137.0,155.6,170.5$.

\section{Results and Discussion}

As part of our efforts to search for novel HDACs inhibitors, we investigated a series of new hydroxamate analogues (A) which were designed as hybrides of TSA/SAHA (Figure 2).

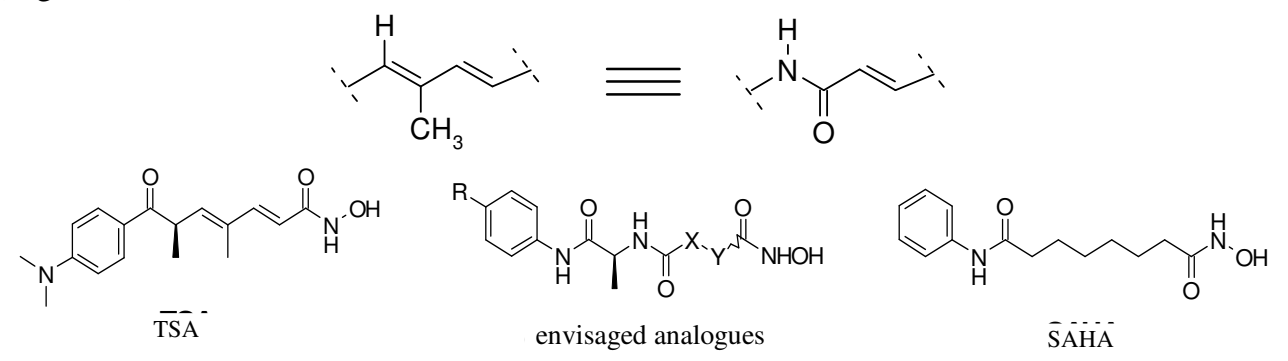

$\mathrm{A}_{1}: \mathrm{X}=\mathrm{CH}_{2}, \mathrm{Y}=\mathrm{CH}_{3}, \mathrm{~A}_{2}: \mathrm{X}=\mathrm{CH}_{1}, \mathrm{Y}=\mathrm{CH}$

Figure 2. Analogues hybrides TSA/SAHA

The rationale for the structure relied on the following parameters. The presence of the amide function on the lateral chain instead of substituted alkene on the TSA, is one common feature shared by these analogues. We can then consider these analogues as TSA isosters. The presence of the carbonyl function on the lateral chain instead of the methyl one, introduces a new hydrophilic feature which can allow the formation of hydrogen binding in the tubular pocket of the enzyme. Such a modification could have an impact on the affinity toward HDACs. These analogues have an aryl recognition moiety which can be substituted in different ways. The orientation of the methyl in the chain is identical to that of TSA. The lateral chain comes from the acylation of an aromatic amine and leads to a pattern which is identical to that of SAHA. This chain will be saturated or unsaturated. In this paper we report the synthesis of these compounds. Scheme 1 shows a general synthetic route of the desired analogues A1-a,b and A2-a.<smiles>[R]c1ccc(NC(=O)C(C)NC(=O)c2ccc(Br)cc2)cc1</smiles> 


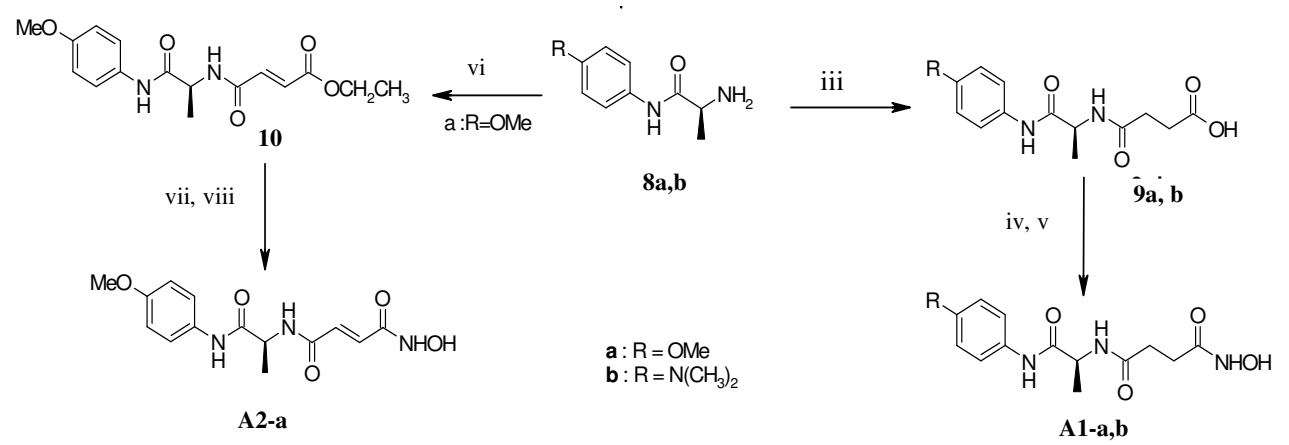

Scheme 1 General synthetic route analogues A1-a,b and A2-a

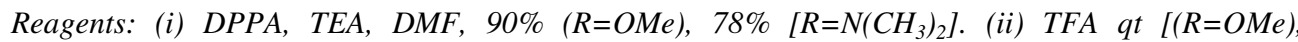
$\left(\mathrm{R}=\mathrm{N}\left(\mathrm{CH}_{3}\right)_{2}\right]$ (iii) anhydride succinic TEA, DMF, 93\% ( $\left.\mathrm{R}=\mathrm{OMe}\right), 61 \%$ [R=N(CH3)2] (iv) $\mathrm{NH}_{2} \mathrm{OBn}$, HCl EDC, HOBT, TEA, DMF,48\% (R=OMe) or DPPA, TEA, DMF 50\% [R=N(CH $\left.\mathrm{CH}_{2}\right](v) \mathrm{H}_{2}, \mathrm{Pd} / \mathrm{C}$, $\mathrm{MeOH}$, qt $(\mathrm{R}=\mathrm{OMe}), 73 \% \mathrm{R}=\mathrm{N}\left(\mathrm{CH}_{3}\right)_{2}$ (vi) EDC, HOBT,TEA, DMF, mono-ethyl ester fumaric acid 49\% (vii) $\mathrm{LiOH}, \mathrm{MeOH} 57 \%$ (viii) $\mathrm{NH}_{2} \mathrm{OTHP}, \mathrm{DMF}, \mathrm{TBTU}, \mathrm{TEA}$.

The $N$-tert-butoxycarbonyl- $L$ - alanine 5 was coupled with para substituted aniline $\mathbf{6 a}, \mathbf{b}$ in several conditions. The use of $N, N$-bis(2-oxo-3-oxazolidinyl)-phosphinique chloride (BOP-Cl) as coupling reagent resulted in low yield $41 \%$. Using DPPA to activate the carboxylic acid gave a $90 \%$ and $78 \%$ yield for respectively $\mathbf{7 a}$ and $\mathbf{7 b}$. The $N$ protecting group was removed after treatment with trifluoroacetic acid. The amine 8a,b reacted in DMF with succinic anhydride in the presence of TEA to give carboxylic acid 9a: 93\%, and 9b: $61 \%$. The resulting acid was converted to the $O$-benzyl protected hydroxamate. Subsequent catalytic hydrogenation of the protected group was used to generate the desired hydroxamates A1-a (qt), A1-b (73\%).

The synthesis of the analogue A2-a started with the coupling of amine 8a with the monoethyl ester fumaric acid (Scheme 1). The ethyl ester $\mathbf{1 0}$ was cleaved under basic conditions in the presence of $\mathrm{LiOH}-\mathrm{MeOH}$ and generated the corresponding acid with $57 \%$ yield. The acid was then converted to the $O$-THP (protected hydroxamate), using TBTU as coupling agent. The hydroxamic acid A2-a was isolated after purification of a small sample on the preparative plates with $66 \%$ yield.

\section{Conclusion}

We have synthesized three analogues hybrides of TSA/SAHA with good yields. From these analogues, some novel synthetic inhibitors can be developed.

\section{References}

1. Suzuki T, NaganoY, Matsuura A, Kohara A, Ninomiya S I, Kohda K and Miyata N, Bioorg Med Chem Lett., 2003, 13(24), 4321-4326.

2. Hanessian S, Auzzas L, Giannini G, Marzi M, Cabri W, Barbarino M, Vesci L and Pisano C, Bioorg Med Chem Lett., 2007, 17(22), 6261-6265.

3. Desai D, Salli U, Vrana K E and Amin S, Bioorg Med Chem Lett., 2010, 20(6), 2044-2047.

4. Maeda T, Nagaoka Y, Kuwajima H, Seno C, Maruyama S, Kurotaki M and Uesato S, Bioorg Med Chem., 2004, 12(16), 4351-4360.

5. Nishino N, Jose B, Shinta R, Kato T, Komatsu Y and Yoshida M, Bioorg Med Chem., 2004, 12(22), 5775-5784. 
6. Jung M, La Thangue N B and Bandara L R, Eds., Humana Press: Totowa, 2002, 123-144.

7. (a) Kelloff G J, Boone C W, Steel V E, Fay J R, Lubet R. A, Crowell J A and Sigman C C, J Cell Biochem., 1995, Suppl. 20, 1-24; (b) Sartorelli A C, Br J Cancer, 1985, 52, 293-302.

8. Coffey D C, Kutko M C, Glick R D, Butler L, M Heller G, Rifkind R A, Marks, P A, Richon V M and La Quaglia M P, Cancer Res., 2001, 61(9), 3591-3594.

9. Wittich S, Scherf H, Xie C, Brosch G, Loidl P, Gerhäuser C and Jung M, J Med Chem., 2002, 45(15), 3296-3309.

10. Monneret C, Eur J Med Chem., 2005, 40, 1-13.

11. Tsuji N, Kobayashi M, Nagashima K, Wakisaka Y and Kiozumi K, Antibiot., 1976, 29(1), 1-6.

12. Yoshida M, Kijima M, Akita M and Beppu T, J Biol Chem., 1990, 265(28), 17174-17179.

13. (a) Richon V M, Emiliani S, Vedin E, Webb y, Bresloww R, Rifkind R A and Marks P A, Proc Natl Acad Sci U S A, 1998, 95, 3003-3007; (b) Bulter L M, Agus D B, Scher H I, Higgins B, Rose A, Cordon-Cordo P, Thaler H T, Rifkind R A, Marks PA and Richon V M, Cancer Res., 2000, 60(18), 5165-5170.

14. Richon V M, Webb Y, Merger R., Sheppard T, Jursic B, Ngo L, Civoli F, Breslow R, Rifkind R A and Marks P A, Proc Natl Acad Sci U S A, 1996, 93(12), 5705-5708.

15. Breslow R, Bel veder S and Gershell L, Helv Chim Acta, 2000, 83, 1685-1692.

16. (a) Remiszewski S W, Sambucetti L C, Atadja P, Bair K W, Cornell W D, Green M A, Howell, K L, Jung M, Kwon P, Trogani N and Walker H, J Med Chem., 2002, 45(4), 753. (b) Jung M, Brosh G, Kölle D, Schef H, Gerhouser C and Loidl P, J Med Chem., 1999, 42, 4669-4679.

17 (a) Kim Y B, Lee K H, Sugita K, Yoshida M and Horinouchi S, Oncogene, 1999, 18(15), 2461-2470; (b) Ohtami, M, Takakaru M, Kazuhira S and Kenji S, J Med Chem., 1996, 39(15), 2871-2873.

18. (a) Suzuki T, Ando T, Tsuchiya K, Fukazawa N, Saito A, Mariko Y, Yamashita T, Nakanishi O, J Med Chem., 1999, 42(15), 3001-3003; (b) Saito A, Yamashita T, Mariko Y, Nosaka Y, Tsuchiya K, Ando T, Suzuki T, Tsuruo T and Nakanishi O, Proc Natl Acad Sci U S A, 1999, 96, 4592-4597.

19. Mai A, Massa S, Ragno R, Cerbara I, Jesacher F, Loidl P and Brosch G, J Med Chem, 2003, 46(4), 512-524.

20. Johnston R W, Nature Rev Drug Discov., 2002, 1, 287-299.

21. Lavoie R, Bouchain G, Frechette S, Hyungwoo S, Abou Khalil E, Leit S, Fournel M, Yan P T, Trachy-Bourget M C, Beaulieu C, Li Z, Besterman J and Delorme D, Bioorg Med Chem Lett., 2001, 11, 2847-2850.

22. Bolden J E, Peart, M J and Johnstone R W, Nat Rev Drug Discov., 2006, 5, 769-784.

23. Saveria M and Pier G P, Nature Rev Cancer, 2006, 6, 38.

24. Glozak M A and Seto E, Oncogene, 2007, 26, 5420.

25. Jose B, Oniki Y, Kato T, Nishino N, Sumida Y and Yoshida M, Bioorg Med Chem Lett., 2004, 4, 5343-5346.

26. Drummond D C, Noble C O, Kirpotin, D B, Guo Z, Scott G K and Benz C C, Annu Rev Pharmacol Toxicol., 2005, 45, 495-528. 


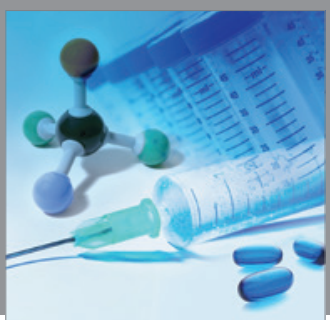

International Journal of

Medicinal Chemistry

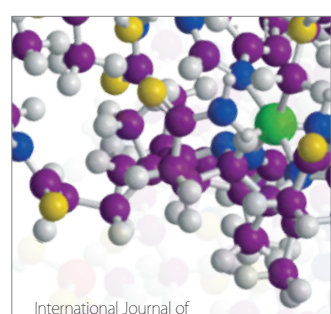

Carbohydrate Chemistry

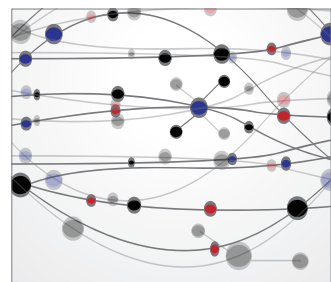

The Scientific World Journal
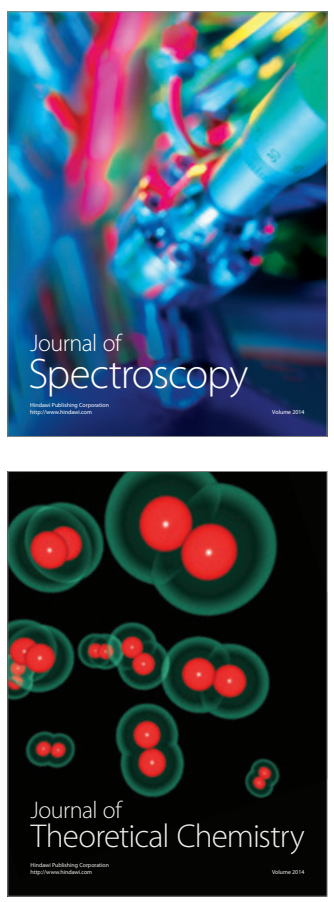
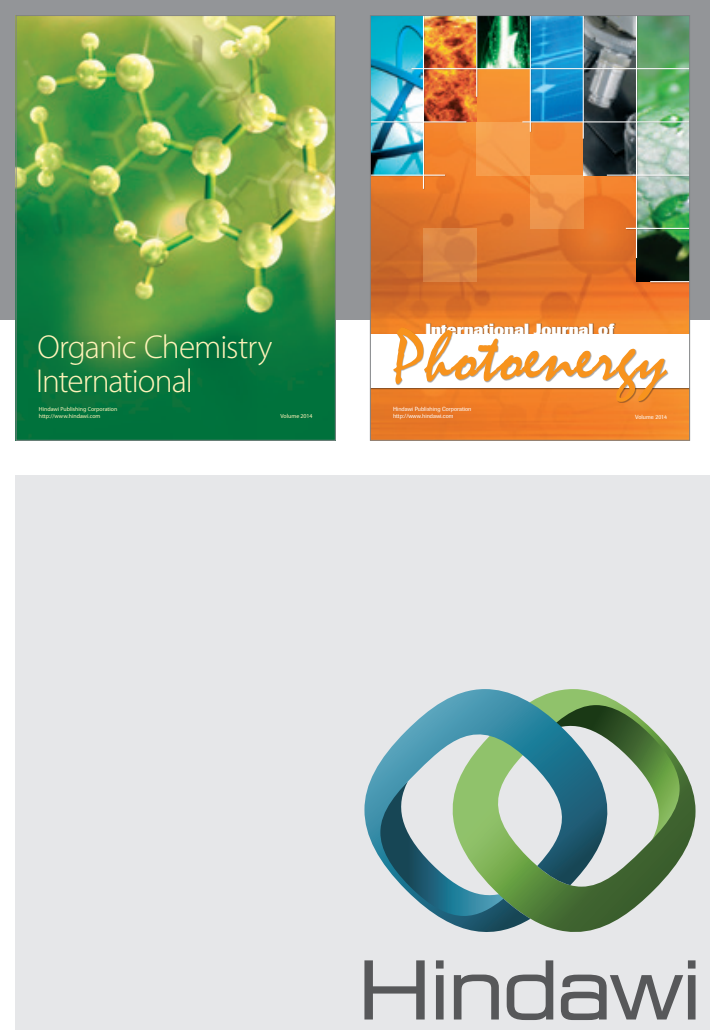

Submit your manuscripts at

http://www.hindawi.com
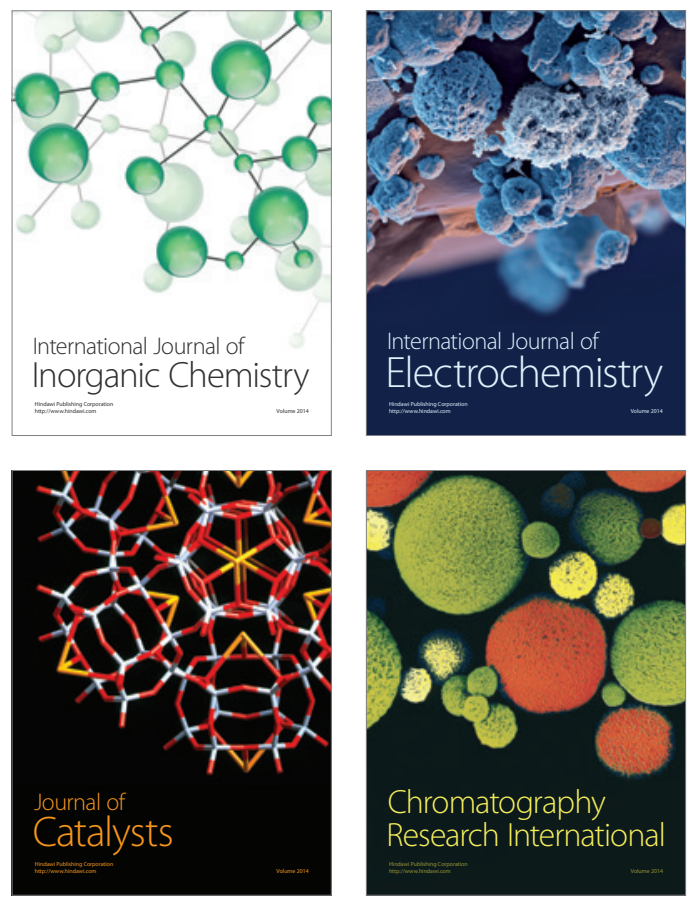
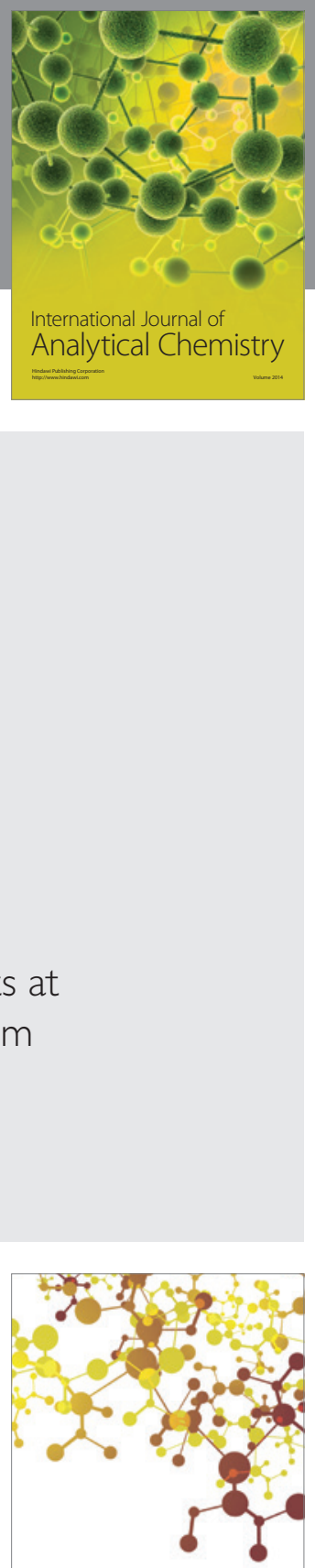

Journal of

Applied Chemistry
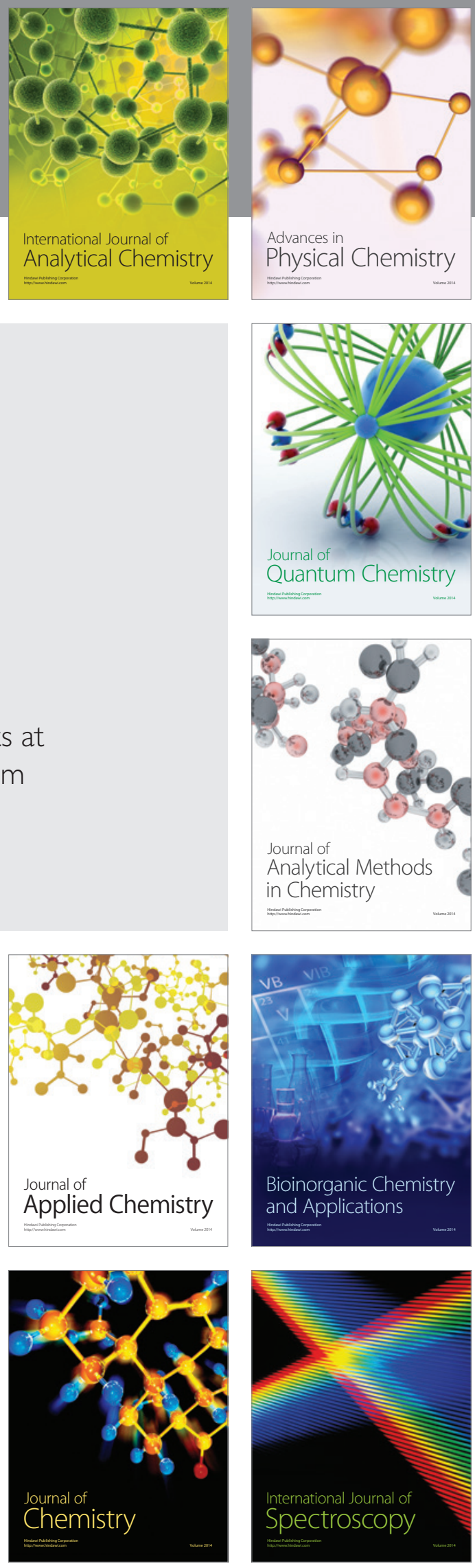